\title{
Field Test Results of an Automated Image Retrieval System
}

\author{
Kenneth W. Tobin*, Thomas P. Karnowski, Lloyd F. Arrowood \\ Oak Ridge National Laboratory ${ }^{\dagger}$, P.O. Box 2008, Bldg. 3500, MS-6010 \\ Oak Ridge, Tennessee 37831-6010 \\ Fred Lakhani \\ International SEMATECH, 2706 Montopolis Dr. \\ Austin, Texas 78741-6499
} \begin{abstract}
monitoring, line width control, and overlay metrology.
The ability to manage large image databases has been a topic of growing research. Imagery is being generated and maintained for a large variety of applications including remote sensing, architectural and engineering design, geographic information systems, and weather forecasting. Content-based image retrieval (CBIR) is a technology that is being developed to address these needs [4]. CBIR refers to techniques used to index and retrieve images from databases based on their pictorial content. Pictorial content is typically defined by a set of features extracted from an image that describe the color, texture and/or shape of the entire image or of specific objects. This feature description is used in CBIR to index a database through various means such as distance-based techniques, approximate nearest-neighbor searching, rule-based decision-making, and fuzzy inferencing $[4,5]$.
\end{abstract}

ABSTRACT

The rapid identification of yield detracting mechanisms through integrated yield management is the primary goal of defect sourcing and yield learning. At future technology nodes, yield learning must proceed at an accelerated rate to maintain current defect sourcing cycle times despite the growth in circuit complexity and the amount of data acquired on a given wafer lot [1]. As integrated circuit fabrication processes increase in complexity, it has been determined that data collection, retention, and retrieval rates will continue to increase at an alarming rate. Oak Ridge National Laboratory (ORNL) has been working with International SEMATECH to develop methods for managing the large volumes of image data that are being generated to monitor the status of the manufacturing process $[2,3]$. This data contains an historical record that can be used to assist the yield engineer in the rapid resolution of manufacturing problems. To date there are no efficient methods of sorting and analyzing the vast repositories of imagery collected by in-line and off-line inspection tools for failure analysis, particle

CBIR addresses a problem created by the growing proliferation of Automatic Defect Classification (ADC) technologies; i.e., the management and use of large amounts of image data. For semiconductor yield management applications we have denoted CBIR technology as Automated Image Retrieval (AIR) [6, 7,

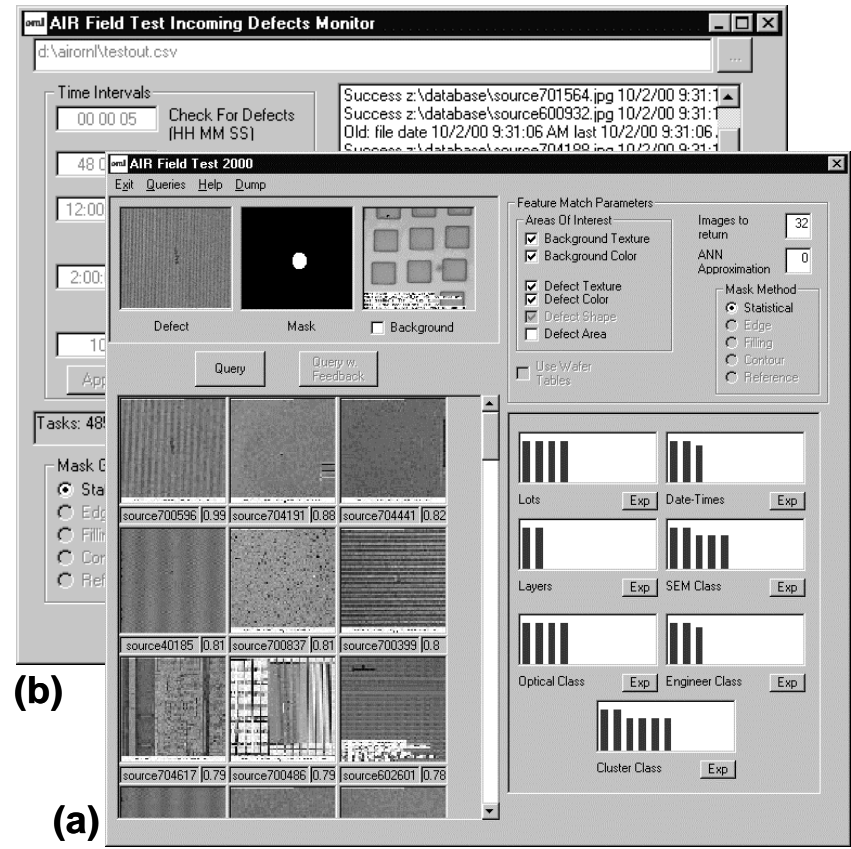

Figure 1 - (a) AIR Field Test software interface for controlling image retrieval. (b) Control panel loading wafer data and images and monitoring progress. 8]. Figure 1 shows an example of a content-based query and result using the ORNL AIR system that was field tested at semiconductor manufacturing sites in the Fall of 2000. Digital imagery for failure analysis is generated between process

\footnotetext{
* K.W.T. (Correspondence): Email: tobinkwjr@ornl.gov; WWW: http://www-ismv.ic.ornl.gov; Telephone: 865-574-8521; Fax: 865-5768380

${ }^{\dagger}$ Prepared by the OAK RIDGE NATIONAL LABORATORY, Oak Ridge, Tennessee, 37831-6285, operated by UT-

BATTELLE, LLC for the U.S. DEPARTMENT OF ENERGY under contract DE-AC05-00OR22725.
} 
steps from optical microscopy and laser scattering systems and from optical, confocal, SEM, AFM, and FIB imaging modalities. This data is maintained in a yield management database and used by fabrication engineers to diagnose and isolate manufacturing problems. The semiconductor industry currently has no direct means of searching the yield management database using image-based queries. Current abilities to query the fabrication process are based primarily on lot number, wafer ID, time/date, process layer, engineer classification, or ADC class, etc. Although this approach can be useful, it limits the user's ability to quickly locate historical examples of visually similar imagery. Without the addition of datamining capabilities such as AIR, this large image repository will remain virtually untapped as a resource for rapidly resolving manufacturing problems.

Image retrieval technologies have been under development since the early 1990's but very few applications have evolved for solving specific, real-world problems. Researchers at ORNL developed the capability for a flexible image retrieval technology for industrial applications that independently takes into account details regarding the product defectivity, substrate, and imaging modality characteristics [7]. The fundamental premise of the AIR technology is that a similar process or phenomena likely generates images that are visually similar[6]. This implies that statistical process information that is associated with the retrieved image list can be used to estimate defect sourcing information.

In this paper we will present yield management integration concepts and the results of initial field-testing completed during the 2000 project year. Examples of query data and results from a semiconductor fabrication environment will be presented and described. Proposed yield management applications of AIR include: providing support of human-level assertions for sourcing manufacturing problems; assisting with the off-line review and analysis of uncommon defects; assisting in the (initial) generation of libraries for ADC systems; providing unsupervised classification of defects during early yield learning; assisting in the training of yield management personnel; and finally, the automated association of a query with a problematic tool or process. Current image retrieval systems for semiconductor manufacturing depend on alphanumeric data to perform retrieval functions (e.g., lot number, time/date, wafer ID, etc.). Without the addition of content-based image retrieval, this large image repository will remain virtually untapped as a resource for rapidly resolving manufacturing problems.

\section{BIOGRAPHY}

Dr. Kenneth W. Tobin leads the Image Science \& Machine Vision Group at the Oak Ridge National Laboratory, Oak Ridge, Tennessee. He has been performing research with the semiconductor industry since 1992 for rapid yield learning that includes image defect classification, wafermap spatial signature analysis, content-based image retrieval, and digital holography. Dr. Tobin is a contributing member of the Semiconductor Industry Association's International Technology Roadmap for Semiconductors in the area of Defect Reduction.

\section{REFERENCES}

[1] Semiconductor Industry Association, International Technology Roadmap for Semiconductors, 1999.

[2] K.W. Tobin, T.P. Karnowski, S.S. Gleason, D. Jensen, F. Lakhani, "Integrated Yield Management", $196^{\text {th }}$ Meeting of the Electrochemical Society, Inc., Oct. 17-22, Honolulu, Hawaii, 1999.

[3] K.W. Tobin, T.P. Karnowski, R.K. Ferrell, and F. Lakhani, "Managing Defect Image Databases for Semiconductor Yield Monitoring and Control", Global Semiconductor, Sterling Publications, February 2000.

[4] V.N. Gudivada and V.V. Raghavan, "Content-Based Image Retrieval Systems”, IEEE Computer Magazine, 0018-9162, September 1995, p. 18.

[5] M. De Marsicoi, L. Cinque, and S. Levialdi, "Indexing Documents by their Content: A Survey of Current Techniques", Imaging and Vision Computing, Vol. 15, 1997, p. 119.

[6] K.W. Tobin, Thomas P. Karnowski, and Fred Lakhani, “The Use of Historical Defect Imagery for Yield Learning”, The $11^{\text {th }}$ Annual IEEE/SEMI Advanced Semiconductor Manufacturing Conference and Workshop, Fairmont Copley Plaza Hotel, Boston, MA, September 12-14, 2000.

[7] K.W. Tobin, T.P. Karnowski, and R.K. Ferrell, "Image Retrieval in the Industrial Environment", IS\&T/SPIE's $11^{\text {th }}$ International Symposium on Electronic Imaging: Science and Technology, San Jose Convention Center, January 1999.

[8] T.P. Karnowski, K.W. Tobin, R.K. Ferrell, and F. Lakhani, "Content Based Image Retrieval for Semiconductor Manufacturing”, IS\&T/SPIE's 12th International Symposium on Electronic Imaging: Science and Technology, San Jose Convention Center, January 2000. 